\title{
Herzog y la ambigüedad significativa
}

\section{Geraldine GERLING}

Universidad Nacional Autónoma de México

Hay libros que leemos cuando somos jóvenes, sobre todo aquellos escritos por autores contemporáneos, que ejercen una gran influencia en nuestras vidas. La época en que ellos vivieron fue nuestra época también; reconocemos los acontecimientos, los espacios, la urdimbre de los eventos como un territorio que nos es familiar, pues el mundo que evocan es, en muchos aspectos, continuo con el nuestro.

Uno de estos libros es Herzog, de Saul Bellow. Considero que en esta novela, más que en ninguna otra escrita por él, Bellow ha logrado explorar las crisis emocionales e intelectuales del hombre moderno en una forma sagaz e imaginativa, pero al mismo tiempo, humorística. Su producción literaria incluye varias novelas, cuentos, obras de teatro y de crítica literaria. Algunas de sus obras más reconocidas son: The Victim (1947), Dangling Man (1944), The Adventures of Augie March (1953), Seize the Day (1956), Henderson the Rain King (1959), Humboldt's Gift (1975) y Mr. Sammler's Planet (1970). Ganó el National Book Award por The Adventures of Augie March en 1953, y por Herzog en 1964, el Premio Pulitzer por Humboldt's Gift en 1975, y el Premio Nobel en 1976.

De hecho, hace poco, cuando se les pidió su opinión, varios escritores y críticos ingleses, entre ellos Martin Amis, Ian McEwan, Malcolm Bradbury, A. S. Byatt y Salman Rushdie, designaron en forma unánime a Saul Bellow como el novelista más extraordinario del siglo XX. Ésta es una anécdota curiosa, pues según Ian Mc Ewan, Bellow era el más europeo de los novelistas norteamericanos. Lo que los escritores británicos encontraban en él, que no podían encontrar entre los suyos era

$[\ldots]$ the generous inclusiveness of his work. Not since the $19^{\text {th }}$ century has a writer been able to render a whole society, without condescension or self-conscious anthropology. Seamlessly, Bellow can move between the poor and their mean streets, and the power elites of university and government, this privileged dreamer with the 'deep-sea thought'. His work is the embodiment of an American vision of plurality. In Britain we no longer seem able to write across the crass and subtle distortions of class — or rather, we can't do it gracefully without seeming to strain or without caricature. Bellow appears larger, therefore, than any British writer can be (Mc Ewan, 2005: 102). 
Cuando el modernismo reemplazó al naturalismo como corriente literaria dominante, una nueva generación de escritores judíos de la posguerra como Bellow, Bernard Malamud y Philip Roth, entre otros, encontraron en las teorías del existencialismo y del psicoanálisis un medio para explorar la influencia de la cultura contemporánea sobre el hombre moderno. En sus novelas, el judío representa al hombre moderno universal: "The Jew became the modern Everyman, everyone's favorite victim, shlemiel ${ }^{1}$ and secular saint" (Dickstein, 2001: 3). No obstante, en Herzog Bellow rompe con lo que él llama "victim literature". "Victim literature purports to show the impotence of the ordinary man. In writing Herzog, I felt I was [...] coming to the end of a literary sensibility [which] implies a certain attitude towards civilization - anomaly, estrangement, the outsider, the collapse of humanism" (Bellow, 1995: 134).

Se ha dicho que la obra de Bellow va a contrapelo de la cultura contemporánea, pues en ella se trastocan ideas o conceptos sobre la realidad comúnmente aceptados por la mayoría. En Herzog se observa una oposición explícita a las ideas de los filósofos existencialistas franceses tales como Sartre o Camus y los nihilistas como Heidegger, Kant o Hegel. Se considera que Bellow fue muy valiente en adoptar una postura contraria a los movimientos filosóficos de su época cuando se opuso a lo absurdo en la literatura, así como al ateísmo de los existencialistas (Miller y Thomas, 1990: 21). Sus ideas son contrarias a la tradición literaria norteamericana de su época, a la cual pertenecen escritores tales como Norman Mailer, Richard Wright, Ralph Ellison, Joseph Heller y John Barth, entre otros, quienes ven al hombre moderno como un ser desplazado por las condiciones de la civilización moderna. El sentido de lo absurdo es una condición mental, casi un modo de vida de sus personajes en una era de gran poder financiero y tecnológico, de cambios caóticos y de desconfianza en las instituciones. A diferencia de los personajes de Mailer y de Wright, por ejemplo, los personajes de Bellow no destruyen a otros para justificarse ni se rinden ante la angustia existencial; más bien, intentan perpetuar las cualidades que los definen como seres humanos - la compasión y el amor a la vida y al prójimo. Asimismo, a través de ellos, Bellow busca descubrir y revelar el yo auténtico que es capaz de trascender la cultura y su autoridad temporal (Pifer, 1990: 1-2). Para Lionel Trilling, la autenticidad es el valor crucial y la fuerza generadora de la literatura moderna: "Striving to achieve authenticity, the spirit must divest itself of all cultural supports and descend in a downward movement through all the cultural superstructures to some place where all movement ends and begins" (Trilling, 1971: 12).

Herzog es no sólo un banquete para el intelecto, sino un viaje por el paisaje de los valores y del pensamiento contemporáneo de la segunda mitad del siglo XX.

\footnotetext{
${ }^{1}$ Palabra en yiddish que se aplica a alguien que maneja determinadas situaciones de la peor manera posible. También se usa para referirse a alguien perseguido por la mala suerte, la cual se debe, en mayor o menor medida, a su propia ineptitud.

Cf. Sanford Pinsker, The Schlemiel as Metaphor. Studies in Yiddish and American Jewish Fiction, p. 2.
} 
Dado que la novela comienza y termina con Herzog en su casa de Ludeyville viviendo entre mariposas, búhos y ratones de campo, algunos críticos en su momento pensaron que Moses Herzog había llegado a un estado de inercia absoluta. Theodore Solotaroff llegó a decir: "One wonders what will happen when the trees of Ludeyville turn bare and Herzog's mind fills again with its dilemmas. God requires more desire than Herzog has to give; so far he hasn't abandoned the old Self but merely found a healing illusion of having done so... The story of his recovery lacks the true opposition of Otherness..." (Solotaroff, 1970: 101).

Por su parte, Norman Mailer criticó a Herzog por ser el único personaje de la novela:

Never has a novel been so successful when its hero was so dim. Not one of the critics who adored the book would ever have permitted Herzog to remain an hour in his house. For Herzog was defeated, Herzog was an unoriginal man, Herzog was a fool..., a sodden fool, overeducated and inept, unable to fight, able to love only when love presented itself as a gift... Herzog was hopeless, we learned nothing about society from him... And he is the only figure in the book. The other characters are seen on the periphery of a dimming vision... One reads the novel with compassion... Herzog inspires sorrow... If we do not feel compassion for him, then we are going to be forced to shoot him (Mailer, 1969: 75).

Por un lado, el enorme éxito de la novela y las opiniones de incontables críticos y lectores son prueba auténtica de que estos ataques fueron inmerecidos. En cuanto a la opacidad de los personajes, se trata de todo lo contrario. Es precisamente dentro de la mente de Herzog donde éstos existen y donde se lleva a cabo la acción. La historia misma se desarrolla dentro de la mente del personaje, plena de emociones variadas y exuberantes.

Por otro lado, lo que estos críticos pasaron por alto es que todo en la vida del personaje es paradójico. Herzog está solo y se aísla de todos, no obstante, siempre insiste en el valor de las relaciones humanas y sueña con el valor de la vida en comunidad; se pronuncia en contra del nihilismo cultural y lo que llama "the Wasteland outlook" (96), pero sufre de una depresión que a veces lo doblega; se enfrasca en una investigación que le pueda proporcionar una explicación sistemática de la realidad, pero su fe en ella se colapsa; se manifiesta a favor del individualismo, pero también lo descarta como una carga indeseable que inhibe el amor al prójimo. Sin embargo, estas y otras paradojas o contradicciones no van en menoscabo de la obra, sino que constituyen una prueba de la ambivalencia que la caracteriza.

Todo comienza con Herzog, que ha estado repasando su pasado en su casa de las montañas Berkshires y dice, "If I am out of my mind, that's all right with me". En este punto Herzog ha llegado casi al fin de su viaje interior, y su estancia en la casa constituye una suerte de marco inicial donde va a recoger sus experiencias del pasado y a componer una visión de ellas tal como existen en su imaginación. Así, desde el principio, el lector tiene un atisbo del "reposo" al que Herzog arribará. Después de este 
inicio premonitorio y por medio de una serie de recuerdos narrados y de monólogos citados, nos enteramos de las etapas sucesivas de las crisis emocionales e intelectuales del personaje. Herzog es un profesor universitario que había iniciado su carrera en forma brillante, pero que ahora ha perdido el rumbo. Está padeciendo lo que Bellow ha señalado como el debilitamiento del hombre culto a causa de las fuerzas condicionantes de la cultura y de la historia (Roudané, 1984: 76). Ha estado escribiendo un libro para analizar el significado social de la Nada y su influencia sobre la condición moderna. Pero a medio camino de su investigación encuentra que ha perdido la fe en las teorías filosóficas de su tiempo. Esta crisis surge durante los meses más sombríos de su matrimonio, pues se ha enterado de que su esposa le había sido infiel mucho antes de su separación, y ahora busca explicaciones que lo puedan proteger de la dolorosa confusión de sus sentimientos. Herzog se encuentra seriamente perturbado y, para su desgracia, Madeleine, su esposa y Gersbach, el amante de ella, se han dedicado a esparcir el rumor de que él es un enfermo mental. Se siente agobiado por la urgencia de encontrar una salida a su predicamento: "He was overcome by the need to explain, to have it out, to justify, to put in perspective, to make amends" (8). Pero la crisis no sólo se debe al golpe moral que ha sufrido, sino que su desdicha lo lleva a darse cuenta de que existen en su entorno social cuestiones no resueltas que su trabajo intelectual abstracto no le pueden ayudar a resolver. Por otra parte, Herzog siente el peso abrumador de su existencia, está descontento con su estado actual y busca reestablecer el orden en su vida: "Until lately, you led a life of innocent sloth. But suddenly a Faustian spirit of discontent and Universal reform descends upon you. Scolding. Invective" (88).

Ahora, es difícil referirse a la vida de Herzog, un personaje lastimado por la soledad, la traición, la desilusión, el dolor y la separación, y al mismo tiempo, señalar que no sólo se trata de una novela donde el personaje explora cuestiones filosóficas profundas, sino que también es una novela cómica e irónica. Bellow mismo en su prólogo al libro The Closing of the American Mind de Alan Bloom señala que le gustaba ridiculizar al norteamericano culto, especialmente en Herzog, que consideraba como una novela cómica en donde se burlaba de la pedantería de los intelectuales y en donde mostraba cuán poco puede ofrecer la "educación superior" a un hombre afligido (Bloom, 1987: 15-16).

Herzog siente que ha caído bajo un "hechizo" que lo mueve a escribir cartas a todo el mundo, a gente famosa, a sus amigos, a sus parientes, a los vivos y también a los muertos, a sí mismo y a Dios. Desea castigar a aquellos que han violado los derechos de la inteligencia humana en el mundo. Lo impulsa su necesidad de justicia para sí y para la humanidad. Las cartas de Herzog son algo verdaderamente extraordinario. Son irreverentes, inspiradas, combativas e irónicas, no sólo a expensas de los demás, sino de sí mismo también. Estas cartas que, paradójicamente, nunca llega a enviar, constituyen una innovación técnica muy atinada. Son una suerte de reductio ad absurdum del género epistolar que, no obstante, sirven a Herzog para hacer observaciones profundas sobre cuestiones filosóficas que le preocupan y también para explorar sus relaciones con los que le rodean. Asimismo, las cartas le dan la oportunidad de dar rienda suelta a su enojo y a expresar su desilusión sobre su vida y su cultura. 
Para Herzog, la realidad tiene dos aspectos fundamentales para los cuales busca conexiones que los puedan explicar. Por un lado, está su vida privada de relaciones fallidas, habitada por personajes tales como Madeleine y Gersbach; por otro, está el mundo de la realidad histórica influida por las ideas de filósofos, pensadores y políticos. Está convencido que, de algún modo, ambos aspectos están conectados y, lo que es más, unos pueden servir como ejemplo de otros. Transforma una y otra vez sus problemas personales en cuestiones universales. Su carta al editor sobre los peligros de la radioactividad es un buen ejemplo: "Mr. Editor, we are bound to be the slaves of those who have the power to destroy us" (68). Herzog obviamente está pensando en su esposa Madeleine cuando escribe esto, pues en otra ocasión escribe: "The strength to do evil is sovereignty" (157). Su necesidad de aclararlo todo se transforma en una necesidad universal: "[People's] great need, their hunger, is for good sense, clarity, truth-even an atom of it. People are dying — it is no metaphor — for lack of something real to carry home when the day is done" (39).

Con su talento para polemizar, Herzog ataca con singular espíritu cómico a los filósofos modernos: A Heidegger le pregunta, "I should like to know what you mean by the expression, 'The Fall of the Quotidian'. When did this fall occur? Where were we standing when it happened?" (65).

Según Tony Tanner, las cartas no sólo representan el intento del personaje por descubrir la verdad, sino que con ellas pretende definir determinadas situaciones ambiguas donde va a contender con los problemas inherentes a estas situaciones, para al final poder aceptar o rechazar una ideología o sistema en particular (Tanner, 300: 1971). Pero para el lector es evidente que esto es algo que Herzog encontrará virtualmente imposible de lograr porque se da cuenta una y otra vez de que no existen respuestas absolutas y cualquiera que sea la determinación que tome, ésta siempre será discutible.

Herzog rechaza con agudeza irónica lo que considera clichés de la enajenación y de la soledad del hombre moderno en una carta que escribe a un colega llamado Shapiro:

Old Proudhon's visions of darkness and evil can't be passed over. But we must not forget how quickly the visions of genius become the canned goods of the intellectuals. The canned sauerkraut of Spengler's Prussian socialism, the commonplaces of the wasteland outlook, the cheap mental stimulants of Alienation, the cant and rant of pipsqueaks about Inauthenticity and Forlornness. I can't accept this foolish dreariness. We are talking about the whole life of mankind (96).

Pero poco después admite que el hecho de pertenecer a una civilización de masas, donde el hombre es transformado por la ciencia, sometido al poder organizado, expuesto a controles enormes, y aislado por el proceso de mecanización del trabajo, significa que carece de una comunidad en sociedad y que, por lo tanto, se encuentra devaluado (247-248). Y luego, en forma paradójica, reconoce que las revoluciones tecnológicas y la transformación vertiginosa de la cultura y los valores han contribuido al progreso mundial (248). 


\section{$120 \square$ HERZOG Y LA AMBIGÜEDAD SIGNIFICATIVA}

Por otra parte, es contradictorio que Herzog haya estado en desacuerdo con los filósofos especulativos, que intentaron construir síntesis para poder aplicarlas a la solución de problemas inherentes a la humanidad, y al mismo tiempo, él mismo haya emprendido la búsqueda de una "gran síntesis", un estudio que debiera culminar con una propuesta para resolver los conflictos del individuo moderno, que le mostrara cómo vivir la vida: "by renewing universal connections, overturning the last of the Romantic errors about the uniqueness of the Self, revising the old Faustian ideology; investigating the social meaning of Nothingness. And more" (53). Y mientras su manuscrito inconcluso yace abandonado en una vieja valija, su colega Mermelstein le gana la idea, "confounding, overwhelming, stunning everyone in the field as Herzog had meant to do" (255). No obstante, Herzog es capaz de asimilar esto y admite que, a diferencia suya, Mermelstein ha sido capaz de darle al mundo un ejemplo de orden y que por esto merece un lugar en la comunidad intelectual. También está convencido de que ha cometido un pecado en contra de sí mismo al lanzarse en busca de esta "gran síntesis", pues con ello ha exagerado su papel en la vida, erigiéndose como el personaje principal. Admite que ha intentado realizarse a expensas de otros intelectuales de su generación y que ha tratado de eclipsarlos creyéndose superior a ellos (255). Ha pecado por exceso de ambición, pero ha fallado por su falta de orden. Como señala su esposa Madeleine: "It's grotesque how disorganized you are. You're no better than any other addict — sick with abstractions" (154). Ha sobreestimado su importancia en el mundo intelectual, haciéndose notar, queriendo ser admirado. Pero también es capaz de burlarse de sí mismo diciendo: "There comes a time when every ridiculous son of Adam wishes to arise before the rest, with all his quirks and twitches and tics, all the glory of his selfadored ugliness, his grinning teeth, his sharp nose, his madly twisted reason, saying to the rest in an overflow of narcissism which he interprets as benevolence - I am here to witness. I am come to be your exemplar! Poor dizzy spook!" (394).

Lentamente Herzog deberá librarse del "sueño del intelecto" y del engaño de las explicaciones totales. Deberá reconocer cómo el pensamiento mismo puede crear un ámbito de confusión y no de claridad como él lo hubiera deseado. "But can thought wake you from the dream of existence? Not if it becomes a second realm of confusion, another more complicated dream, the dream of intellect, the delusion of total explanations" (206). Queriendo abarcar demasiado, se ha engañado; se siente invadido por una sensación de impotencia, de fracaso y de desprecio hacia sí mismo. El dolor se ha convertido en el centro de gravedad de su existencia y siente que la enajenación es el resultado de sus elucubraciones. Su confusión emocional lo lleva a fustigarse por lo que considera una pasividad conformista de su parte. Sin embargo, no es insensible al humor de su conducta masoquista, y se increpa con sorna: "Loathsome! O patient Griselda Herzog!" (83).

Reconoce que le había conferido un valor positivo al sufrimiento y al dolor como una forma de vida más intensa, como preludio a un despertar verdadero y como un antídoto contra lo ilusorio, pero admite que ha ido demasiado lejos porque el sufrimiento sólo le ha abierto el camino para sentir lástima por sí mismo. Aún estando agobiado por 
estos sentimientos, Herzog se rehúsa a hundirse "down in the mire of post-Renaissance, post-humanistic, post-Cartesian dissolution, next door to the Void" (118). Se pronuncia en contra del nihilismo cultural del siglo XX y en contra de la desvalorización de la vida humana en la sociedad moderna. Se siente hastiado por aquellos que están convencidos de la existencia del infierno y del castigo eterno, mientras ellos disfrutan una vida cómoda y segura.

Vislumbra una salida de su laberinto mental cuando decide posponer su búsqueda de una definición de la naturaleza humana al reconocer que la investigación científica no se ocupa en buscar definiciones. "Modern science, least bothered with the definitions of human nature, knowing only the activity of investigation, achieves its profoundest results through anonymity, recognizing only the brilliant functioning of the intellect" (161). Cae en la cuenta de que el no pensar no es algo fatal por necesidad, pero, al contrario, poco después debe admitir que "the human intellect is one of the great forces of the universe. It can't safely remain unused" (379). La paradoja medular en la experiencia de Herzog es que sólo le es posible renovar su conexión con el resto del mundo a través de su yo interno, pero su tendencia a involucrarse en forma excesiva con su yo puede poner en peligro esta conexión.

Cuando Herzog deja de sentir compasión por sí mismo y se libera de la amargura que le había invadido, deja de culpar a Dios y a los hombres de su infortunio. Siente que le ha sido revelada una verdad fundamental y cita algunas frases del poema "The Four Zoas" de Blake (133.24): "brotherhood is what makes a man human... 'Man liveth not by Self alone but in his brother's face... Each shall behold the Eternal Father and love and joy abound'... The real and essential question is one of our employment by other human beings and their employment by us. Without this true employment, you never dread death, you cultivate it" (333).

La novela termina con la negativa del protagonista a someterse nuevamente a una existencia intelectual pero vacía. El papel que asumirá dentro de la sociedad permanece ambiguo, pues se trata de un primer paso hacia la libertad para elegir lo que es para él, entre muchas posibilidades, la solución más viable para vivir una vida plena, y esto no constituye algo trivial. Ha aprendido que el intelecto que confina su pensamiento a la vida privada se somete a las limitaciones que ese aislamiento le aporta y se percata al fin de que ser un intelectual en estas condiciones no es un privilegio, sino otra forma de esclavitud.

En la mente de Herzog se ha librado una batalla incesante entre ideas que chocan entre sí, pero la contienda más crítica es aquella que se libra en el interior de éstas, en las paradojas y contradicciones que le impiden obtener la claridad de juicio y la iluminación que tanto anhela. Por ello, es el conflicto lo que resulta de capital importancia en la novela. Lo que cuenta no es que Herzog descubra una verdad esencial, sino que persevere en su búsqueda; no es la solución a determinados problemas lo que debe lograr, sino el compromiso perpetuo con su búsqueda; no se trata de que alcance la iluminación a la que aspira, sino que tenga fe en que existe la posibilidad de lograrlo, de modo que su intelecto e imaginación sigan esforzándose al máximo. Con todo 
esto, lo que Herzog descubre es que la intensidad de la búsqueda es lo que da sentido a la vida humana. "And this is the unwritten history of man, his unseen, negative accomplishment, his power to do without gratification for himself, provided there is something great, something into which his being, and all beings can go. He does not need meaning as long as such intensity has scope. Because then it is self-evident; it is meaning" (353) (las cursivas son mías).

Al final de la novela reconoce que sólo lo incomprensible puede iluminar el intelecto humano: "Go through what is comprehensible, and you will see that only the incomprehensible gives any light" (325).

El hecho de que el sentido, el significado último de la vida casi nunca sea unívoco ni claro, que la iluminación se desvanezca casi al momento en que se manifiesta al ser humano, que la verdad sea posible e imposible al mismo tiempo, todo ello aumenta la intensidad de la búsqueda, pero al mismo tiempo impide encontrar soluciones o llegar a conclusiones que pudieran cancelar dicha búsqueda.

Es evidente que hay un propósito detrás de la oscuridad aparente en la novela. La ambigüedad y la paradoja son aspectos ineludibles de la vida misma y su presencia en distintas situaciones de la novela cautiva la atención del lector. La promesa de que existe una respuesta posible lo seduce y lo obliga a atender a los detalles concretos de la búsqueda imaginativa y le exige una participación intensa en ella.

\section{Obras citadas}

Bellow, Saul. 1995. Citado por David D. GallowaY en "Moses-Bloom-Herzog: Bellow's Everyman". The Critical Response to Saul Bellow. Gerhard BACH, ed. Westport: Greenwood Press. 1987. Prólogo a The Closing of the American Mind de Allan BLoom. Nueva York: Simon \& Schuster. . 1965. Herzog. Greenwich, Conn: Fawcett Publications.

BLoom, Allan. 1987. The Closing of the American Mind. Nueva York: Simon \& Schuster.

Dickstein, Morris. 2001. "Never Goodbye Columbus: The Complex Fate of the Jewish-American Writer". The Nation, vol. 273. Oct. 22

Erdmann, David V., ed. 1988. "Vala, The Four Zoas, Night the Ninth, Being the Last Judgment". The Complete Poetry and Prose of William Blake. Nueva York: Random House.

MAILER, Norman. 1969. “The Argument Reinvigorated”. The American Novel since World War II. Marcus KLEIN, ed. Greenwich, Conn: Fawcett Publication.

MC EWAN, Ian. 2005. The Guardian, 7 de abril. Citado en The Wilson Quarterly, vol. 29, Issue 3, verano de 2005. "Paying Tribute to Mr. Bellow: A Survey of Recent Articles". 
MiLler, Eugene G. y Edmund J. ThOMAS. 1990. Writers and Philosophers; A Sourcebook of Philosophical Influences on Literature. Nueva York: Greenwood Press.

PIfER, Ellen. 1990. Saul Bellow Against the Grain. Filadelfia: University of Pennsylvania Press.

PINSKER, Sanford. 1991. The Schlemiel as Metaphor. Studies in Yiddish and American Jewish Fiction. Carbondale: Southern Illinois University Press.

RoudAné, Matthew. 1984. "An Interview with Saul Bellow”. Contemporary Literature, 25, otoño.

SolotarofF, Theodore. 1970. The Red Hot Vacuum and other Pieces of the Writing of the Sixties. Nueva York: Atheneum.

TrILling, Lionel 1971. Sincerity and Authenticity: The Charles Eliot Norton Lectures, 1969-1970. Cambridge, MA: Harvard, UP. 